\title{
Ciudades sustentables. De la gestión a la valoración institucional de las áreas verdes y el arbolado: conurbación Temuco-Padre las Casas

\author{
Sustainable Cities. From management to institutional valuation of
} urban trees and green spaces: Temuco-Padre Las Casas conurbation
}

\author{
Tomás Pastene Reyes ${ }^{1}$, Alfonsina Puppo Stuardo ${ }^{1}$
}

\begin{abstract}
Resumen
Este artículo es parte de una investigación que analiza los vínculos y operaciones de las instituciones encargadas de la gestión de la vegetación urbana en las ciudades: Temuco y Padre Las Casas. Para ello, se trabaja en base a la construcción de un modelo de análisis que permite identificar las tensiones y los nodos problemáticos de ambas ciudades, a partir de los conceptos de: planificación, integración, adaptación y legitimación. Se utiliza el concepto de servicios ecosistémicos a un nivel operativo, ya que permite asimilar el modo en que las instituciones locales pueden comprender los beneficios directos o indirectos que provee el ecosistema urbano al bienestar humano y a las condiciones socioambientales del territorio. Los municipios cuentan con una naturaleza jurídica dual: son organismos de administración del Estado, con capacidades autónomas de gestión. Esta doble capacidad se convierte en un mecanismo que entorpece las dinámicas territoriales y urbanas para un adecuado manejo de la vegetación en ambas comunas.
\end{abstract}

Palabras Clave: ecosistemas urbanos, servicios ecosistémicos, planificación urbana, gestión local.

\begin{abstract}
This article is part of an investigation that aims to analyzing institutional links and operations for the management of urban vegetation in Temuco and Padre Las Casas cities. The research is based on an analysis model that identifies tensions and problematic nodes in both cities, applying concepts of planning, integration, adaptation and legitimation. Ecosystem services are used as a concept that helps institutions to understand and to identify direct or indirect benefits that the urban ecosystem provides for human well - being and the socio - environmental conditions of the territory. Legally, Chilean municipalities have a dual core: they are State administration entities, with autonomous management capacities. The overlap of those qualities becomes a mechanism that stuck the territorial and urban dynamics for adequate management of urban vegetation.
\end{abstract}

Keywords: urban ecosystems, ecosystem services, urban planning, local management.

Recibido el 03 de julio de 2017, aceptado el 27 de octubre de 2017.

1 Laboratorio Ecosistemas Urbanos, Facultad de Agronomía e Ingeniería Forestal, Pontificia Universidad Católica de Chile. Avda. Vicuña Mackenna 4860. Santiago. Email: alfonsina.puppo@uc.cl 


\section{Introducción}

Para avanzar en el desarrollo de ciudades sustentables en Chile, este artículo presenta parte de una investigación que analiza los vínculos y operaciones de las instituciones encargadas de la gestión, el manejo y la mantención de áreas verdes y el arbolado urbano en las comunas Temuco y Padre Las Casas, durante el año 2017. No obstante, el problema conceptual detrás de ese objetivo es, cómo generar mecanismos de gestión, vínculos estratégicos, planes y políticas de regulación en las instituciones públicas que apunten a la sustentabilidad del territorio y, cómo hacer que las instituciones públicas tengan la capacidad de abordar el desarrollo sustentable de las ciudades con una visión integral sobre los procesos de gestión, manejo y mantención de la vegetación y el paisaje urbano. Estas preguntas nacen de la información recolectada en terreno, durante enero 2017, en Temuco y Padre Las Casas, a través de 33 entrevistas en profundidad a actores del sector público que participan en el proceso de gestión, manejo y mantención de áreas verdes y el arbolado urbano, de ambas comunas $\mathrm{y}$, de la revisión de normativas, documentos institucionales y programas que regulan, influyen y disponen recursos para el desarrollo de ese proceso en el territorio.

Los aportes del estudio van orientados a profundizar en la dimensión social del desarrollo sustentable de las ciudades chilenas, a través de un análisis a los procesos institucionales que intervienen en la gestión, el manejo y la mantención de la vegetación urbana, (Dempsey, Bramley, Power y Brown, 2011). Se utiliza el concepto de servicios ecosistémicos a un nivel operativo, ya que permite asimilar el modo en que las instituciones locales pueden comprender los beneficios directos o indirectos que provee el ecosistema urbano al bienestar humano y a las condiciones socio-ambientales del territorio (Laterra, Martín-López, Mastrángelo y Garibaldi, 2017; Gómez-Baggethun, et al., 2013;
Balvanera \& Cotler 2007). Bajo esa lógica, se evalúa la aplicabilidad del concepto en la planificación urbana, en las actividades de gestión y aprovechamiento de los recursos y servicios ambientales para el bienestar humano de la población que habita la conurbación Temuco-Padre Las Casas.

La sustentabilidad de las ciudades es resultado del balance socio-ecológico que alcanzan las interacciones entre los distintos componentes del ecosistema urbano, por eso el análisis que se propone aborda la planificación urbana y las acciones institucionales de manejo ambiental en el caso de estudio desde un enfoque diacrónico y multidisciplinario, para trabajar de manera integral las interacciones emergentes en el ecosistema urbano, (Laterra et al., 2017; Vásquez, 2016; Gómez-Baggethun et al., 2013). Estos procesos se analizan desde una perspectiva sociológica que identifica la red institucional de gestión y manejo de las áreas verdes y el arbolado urbano en Temuco y Padre Las Casas, vinculando los datos del territorio con las acciones institucionales que influyen en el desarrollo del ecosistema urbano. El enfoque diacrónico del análisis abarca los procesos, operaciones y vínculos que modifican o impactan los niveles de sustentabilidad ecológica del territorio a mediano y largo plazo (Caro-Caro \& Torres-Mora 2015; Grimm, Grove, Pickett y Redman, 2000).

Los datos del estudio se ordenan conceptualmente a través de un modelo de análisis ad-hoc que permite integrar las problemáticas emergentes en el territorio. El análisis de las interacciones muestra las brechas y oportunidades que presentan las instituciones para incorporar criterios ecológicos asociados al concepto de servicios ecosistémicos en sus procesos de gestión, manejo y mantención de las áreas verdes y el arbolado urbano (Rusch, Rusch, Goijman, Varela y Claps, 2017).

Los servicios ecosistémicos se trabajan como una categoría de análisis cualitativo 
y cuantitativo, pero también, como una herramienta conceptual y metodológica, porque de esa manera permiten evaluar los alcances y capacidades de incorporar criterios ecológicos en las operaciones y objetivos institucionales del territorio (Gómez-Baggethun et al., 2013). A nivel conceptual, muestran los rendimientos positivos del ecosistema en el bienestar humano y eso hace posible incorporar criterios ecológicos en las acciones y decisiones institucionales relativas a las áreas verdes y el arbolado, traduciendo la visión ecológica en metas institucionales objetivas (Rusch et al., 2017). A nivel metodológico, permite levantar un diagnóstico sobre la cuantificación y localización de los servicios ecosistémicos en el territorio, mostrando los beneficios que proveen las condiciones ambientales al bienestar humano.

El análisis mixto relaciona ambos niveles vinculando la dimensión social y la dimensión ambiental del desarrollo sustentable en las ciudades chilenas mediante el reconocimiento de los beneficios directos e indirectos que provee el ecosistema urbano al bienestar de la población humana, distinguiendo aquellos servicios ecosistémicos que están siendo manejados por las instituciones y aquellos que aún no han sido incorporados dentro de la gestión de áreas verdes y arbolado urbano. La relación conceptual de los datos se apoya analíticamente en la teoría fundamentada (Glaser \& Strauss, 1967), técnica de análisis de datos cualitativos propia de las ciencias sociales que permite vincular información de distinta naturaleza alrededor de un fenómeno social específico (Charmaz, 2006). En este caso, el eje de relación es el proceso de gestión, manejo y mantención de la vegetación urbana de Temuco y Padre Las Casas. Como resultado, se obtiene una visión integral sobre la influencia que tienen las instituciones del territorio (dimensión social) en el desarrollo sustentable del ecosistema urbano (dimensión ambiental) (Dempsey et al., 2011).
Esta investigación se enmarca en un proyecto que busca traducir $\mathrm{y}$ operacionalizar indicadores cuantitativos sobre el aprovisionamiento de servicios ecosistémicos que presenta la conurbación Temuco-Padre Las Casas en indicadores cualitativos que sean útiles a la gestión, el manejo y la mantención institucional de los servicios ecosistémicos del territorio (FONDECYT N 1161709, 2016-2019). No obstante, el artículo presenta solamente los resultados preliminares del análisis de datos cualitativos que se realizó de las entrevistas en profundidad a los actores institucionales vinculados a la vegetación urbana de ambas comunas.

\section{Metodología}

El objetivo del estudio es analizar las operaciones y vínculos que articulan los procesos de gestión, manejo y mantención de la vegetación urbana en Temuco y Padre Las Casas, para reconocer las brechas y oportunidades de incorporar el concepto de servicios ecosistémicos en la red institucional encargada de esas operaciones en el territorio. Las acciones y decisiones institucionales se contrastan con los aportes que entrega el concepto de servicios ecosistémicos y los criterios ecológicos asociados a éste en materia de manejo y mantención de la vegetación urbana (Gómez-Baggethun et al., 2013). En ese marco, el estudio se concentra en los discursos, acciones y decisiones institucionales sobre la gestión, manejo y mantención de la vegetación urbana en la conurbación.

Las fuentes de información utilizadas en el estudio son: a) documentos institucionales e instrumentos de planificación urbana que influyen en el proceso de gestión, manejo y mantención de la vegetación urbana en la conurbación Temuco-Padre Las Casas; b) entrevistas en profundidad (33) realizadas durante enero 2017 a los actores clave de las instituciones que se vinculan con las áreas verdes y el arbolado urbano de Temuco y Padre Las Casas. 
Los documentos institucionales se utilizan para comprender el marco normativo y los programas estatales que regulan la red institucional asociada a la vegetación urbana local y las entrevistas en profundidad se utilizan para ahondar en las operaciones y vínculos inter e intra institucionales que mantienen activa la red institucional de actores encargados de la vegetación urbana en la conurbación. El análisis comparado entre ambas fuentes de información permite identificar el alcance de las funciones que cumplen los actores a lo largo del proceso institucional de gestión, manejo y mantención de las áreas verdes y el arbolado urbano. La orientación de los actores da cuenta de las estrategias y el sentido que orienta a la red institucional en el territorio, sea a nivel individual, de institución o de alianzas estratégicas emergentes en el proceso. En distintos niveles organizacionales, las operaciones y vínculos que se realizan en el territorio permiten analizar: i) las metas individuales de cada actor; ii) los objetivos internos de la institución que representan; y iii) los objetivos que comparte la red institucional de actores encargados de la vegetación urbana.

La selección de entrevistados se definió bajo criterios cualitativos, distinguiendo el tipo de entrevistado y el nivel de información alcanzado sobre las operaciones y vínculos que activan la red institucional de la vegetación urbana local: i) deben ser actores clave de la red institucional de la vegetación urbana local: los entrevistados deben poseer grados de participación e influencia en el proceso de gestión, manejo y mantención de las áreas verdes de Temuco y Padre Las Casas, y ii) deben abarcar la máxima cobertura informativa sobre la red institucional de la vegetación urbana local (saturación de información): Reiteración de datos sobre el proceso de gestión, manejo y mantención de las áreas verdes de Temuco y Padre Las Casas. Ambos criterios aseguran la máxima cobertura de datos cualitativos a nivel de red institucional vinculada a las áreas verdes y el arbolado urbano de estas comunas.

El análisis de la información permite obtener una imagen de la visión general que tiene, la red institucional local, sobre la vegetación urbana, distinguiendo los intereses particulares de cada actor e institución involucrada, así como también, los temas que impactan en la agenda medioambiental del territorio. El contraste entre las declaraciones de los entrevistados con las operaciones y los vínculos que manejan al interior de la red institucional da cuenta de sus capacidades particulares de cobertura, en términos materiales, económicos, normativos, políticos, etc., $\mathrm{y}$ de las funciones que cumplen a lo largo del desarrollo de la gestión, manejo y mantención de la vegetación urbana en la conurbación. Esas funciones aparecen como una categoría de análisis de segundo orden, porque se sostienen de las relaciones entre los datos y desde ahí, permiten explicar el alcance de las operaciones y los vínculos institucionales que se desarrollan en el territorio. En esas relaciones se observan las tensiones, brechas y discontinuidades que emergen durante el desarrollo de las intervenciones institucionales asociadas a la vegetación urbana y a su vez, dan cuenta de la estructura orgánica que sostiene y activa los vínculos al interior de la red institucional, más allá incluso de lo que está establecido en los documentos institucionales que regulan el proceso de intervención.

La red de relación entre los datos se elabora a partir de nodos que conectan los temas del análisis. Estos nodos son los vínculos y operaciones que realizan los actores de la red institucional para llevar adelante el proceso de gestión, manejo y mantención de la vegetación urbana. A nivel micro y macro, dan cuenta de las capacidades y limitantes institucionales con que opera la red institucional en el territorio, mostrando las brechas y oportunidades que existen en cada institución para incorporar criterios ecológicos en sus planes y estrategias de 
manejo de las áreas verdes y el arbolado urbano. Los vínculos y acciones de la red institucional articulan múltiples recursos y estrategias públicas, los cuales, a su vez, dan cuenta de los intereses que persiguen los programas estatales, como también, la orientación estratégica y política detrás de los instrumentos de planificación y ordenamiento territorial que impactan en el desarrollo del ecosistema urbano. A partir de eso, se contrastan las capacidades institucionales locales con la regulación y los programas nacionales para mostrar las brechas que existen al interior del Estado en materia de manejo y mantención de las áreas verdes y el arbolado.

Teoría Fundamentada: construcción de modelos ad-hoc en el análisis de datos cualitativos

Es una tradición metodológica $\mathrm{y}$ de análisis cualitativo de ciencias sociales que relaciona de manera sistemática los datos empíricos, sin considerar elementos externos a los fenómenos estudiados, con el objetivo de generar categorías conceptuales que permitan comprender los fenómenos sociales estudiados (Glaser, 2002). La recolección de datos y el análisis de la información se realizan de manera sistemática y complementaria, mediante un proceso de comparación constante entre los datos. El proceso de investigación avanza estableciendo hipótesis y comparando esas reflexiones con los datos emergentes en el terreno, para corroborar, afinar y darle una mayor capacidad de cobertura a los modelos explicativos que se generan para comprender fenómenos específicos, utilizando únicamente los datos $\mathrm{y}$ variaciones observadas en terreno. Los ajustes del análisis se generan a partir de la relación y reiteración sistemática de datos, lo cual permite distinguir y establecer determinados órdenes y jerarquías semánticas al interior de los modelos explicativos. La cercanía de la relación que se establece con los elementos que componen el fenómeno estudiado evita cualquier interpretación externa al caso analizado, ya que el objetivo es obtener una explicación ad-hoc que se sostenga exclusivamente en los datos sin la necesidad de recurrir a fuentes externas (TrinidadRequena, Carrero-Planes, Soriano, 2006).

La operación básica del análisis es relacionar y comparar todos los insumos informativos hasta construir una red semántica de datos que alcanza la máxima cobertura de información sobre el fenómeno estudiado (Padilla Beltrán et al., 2014). En el análisis de datos cualitativos, la comparación muestra la variabilidad y las particularidades de los fenómenos estudiados: "El análisis comparativo es un rasgo esencial de la investigación de las ciencias sociales (...) Cada incidente se compara con otros, en cuanto a sus propiedades o dimensiones (...), en busca de similitudes y diferencias, y se ubica en una categoría (...) las comparaciones teóricas se pueden derivar de la literatura y la experiencia. No es que usemos la experiencia o la literatura como datos, sino que usamos las propiedades y dimensiones derivadas de los incidentes comparativos para examinar los datos que tenemos frente a nosotros. Así como no reinventamos cada día el mundo que nos rodea, en el análisis nos basamos en lo que sabemos para que nos ayude a comprender lo que ignoramos. Las comparaciones teóricas son herramientas (una lista de propiedades) para observar algo con alguna objetividad más bien que darle un nombre o clasificarlo sin un examen profundo del objeto en cuanto a sus propiedades y dimensiones" (Strauss \& Corbin, 2002 p.86).

El método de comparación constante, aplicado en el análisis de teoría fundamentada permite elaborar modelos explicativos ad-hoc a los casos de estudio, considerando las propiedades, similitudes y diferencias internas, $y$ externas entre los distintos fenómenos que aborda la investigación. Los modelos se construyen a partir de categorías de análisis mediante la codificación, clasificación y 
conceptualización de los datos recolectados en terreno. El ordenamiento teórico de los datos y categorías de análisis da forma al fenómeno de estudio y se estructura en base a las relaciones observadas en terreno (Flores, 2009; Charmaz, 2006; Strauss \& Corbin, 2002). Al reconocer la estructura semántica del fenómeno estudiado, se justifica su emergencia en el entorno a través de un modelo teórico, que permite explicar de manera coherente su desarrollo $\mathrm{y}$ las condiciones bajo las cuales se reproduce. Ese modelo teórico es resultado de la asociación y comparación sistemática de los datos hasta llegar a la saturación de la información (Flores, 2009; Charmaz, 2006).

En este caso, se refieren a la relación $y$ el contraste de las entrevistas en profundidad, con los documentos institucionales revisados, con el fin de abordar todas las operaciones y vínculos del proceso institucional sobre la gestión de la vegetación urbana de Temuco y Padre Las Casas. Cuando se detecta una reiteración sistemática de los datos, se asume una saturación de la información recolectada en terreno. Eso permite generar una explicación teórica del fenómeno social estudiado, que comprende todas las condiciones, posibilidades y propiedades específicas del caso. Las respuestas de los entrevistados y los documentos institucionales revisados se asocian analíticamente para identificar los vínculos, operaciones y procesos que realiza la red institucional en materia de vegetación urbana y sus alcances con el concepto de servicios ecosistémicos. Las interacciones y tensiones entre los actores y los reglamentos institucionales permiten observar por dentro y por fuera el circuito de los actores que participan y se vinculan con las áreas verdes y el arbolado urbano del territorio, distinguiendo las brechas y oportunidades para incorporar el concepto de servicios ecosistémicos y criterios ecológicos en las operaciones de gestión. La justificación de la técnica de análisis utilizada, así como la coherencia interna entre los datos que se analizan, dependen del modelo explicativo que articula el informe. Este instrumento teórico es el centro de la investigación, ya que permite operacionalizar los datos cualitativos y convertirlos en categorías de análisis, optimizando la capacidad explicativa del estudio frente a un proceso social específico. Esta herramienta permite observar inductivamente la información de la red institucional e identificar los principales temas asociados a las áreas verdes y el arbolado urbano. Se comprende así, la emergencia de procesos y dinámicas en el entorno, a partir de los vínculos y operaciones institucionales asociados a la vegetación urbana de la conurbación.

Los modelos de análisis ad-hoc permiten establecer relaciones semánticas entre las distintas instituciones, a partir del cómo conciben los servicios ecosistémicos y cuáles son los criterios ecológicos que incorporan en la gestión de sus políticas, programas y proyectos. A nivel metodológico, el vínculo entre campos semánticos permite traducir los criterios ecológicos a un código institucional; a nivel conceptual, hace posible identificar las brechas y oportunidades para incorporar el concepto de servicios ecosistémicos en la red institucional del territorio. La codificación de datos y significados requiere de un sustento teórico que dé sentido a los temas emergentes en la investigación. Esa base conceptual se genera a través de modelos de análisis adhoc, porque permiten integrar de forma coherente los datos que se superponen entre los distintos campos semánticos que aborda el fenómeno de estudio; del mismo modo, justifican teóricamente el análisis porque se basan en la referencia empírica de los datos. Estos modelos, además de ser explicativos respecto del fenómeno estudiado, establecen relaciones entre los datos a partir de un sustento empírico y así aseguran la existencia sustantiva de los vínculos y procesos que se analizan. 


\section{Procesamiento de la información con el uso de software Atlas.Ti (v.7)}

El ordenamiento de los datos, la operacionalización de categorías y subcategorías de análisis y la construcción del modelo de análisis se apoya en el uso de Atlas.Ti (v.7), software especializado en el análisis de datos cualitativos. Es una herramienta computacional muy eficiente y pertinente al trabajo con redes complejas y análisis semánticos basados en la teoría fundamentada, ya que facilita la labor de clasificación, selección, comparación, vinculación y elaboración de modelos ad-hoc a partir de datos cualitativos de investigación social.

La transcripción de las entrevistas, fueron procesadas computacionalmente en Atlas. ti (v7), generando la codificación de la información y levantando las categorías de análisis de segundo orden. Al relacionar las categorías y los códigos emergentes, se comenzó a elaborar el modelo de análisis al caso, recurriendo a la construcción de redes semánticas y visualizaciones analíticas que permitían representar gráficamente, los vínculos y operaciones que sostienen la red institucional sobre la gestión de la vegetación urbana en Temuco y Padre Las Casas. El uso del software permite sintetizar la información compilada en el estudio, mediante la asociación y distinción de diferencias conceptuales y de la clasificación de los campos semánticos que abordan el proceso institucional frente a estas temáticas (Padilla Beltrán et al., 2014).

Modelo de análisis al caso de estudio: conurbación Temuco-Padre las Casas

El modelo de análisis establece un escenario de posibilidades para incorporar el enfoque de los servicios ecosistémicos en la planificación urbana. Ese escenario se utiliza de contraste al estado actual del territorio para evaluar el alcance y los impactos de las operaciones que realiza la red institucional de la vegetación urbana local. Los procesos y decisiones institucionales en el territorio están mediados por factores externos de distinta naturaleza y escala, por ende, el análisis de las operaciones y vínculos que activan esta red institucional, deben realizarse mediante un instrumento conceptual que indique los alcances y el impacto de estos procesos de gestión. Bajo esa lógica, se deben establecer relaciones funcionales entre los distintos componentes, para que el modelo se vuelva dinámico e integre los procesos emergentes del territorio en el desarrollo del ecosistema urbano.

En el marco de la teoría fundamentada, los componentes del modelo son categorías de análisis que tienen la máxima capacidad de cobertura semántica sobre los temas relativos al objeto de estudio, es decir, ecosistema, entorno construido, comunidad e instituciones. Cada uno de esos componentes, dice relación con una dimensión específica del ecosistema urbano: i) Ecosistema: infraestructura y elementos verdes del paisaje; emplazados en los entornos construidos o ciudades; ii) Entorno Construido: infraestructura urbana y elementos grises del paisaje; entorno urbano y periurbano; iii) Comunidad: prácticas, usos y demanda social de la población humana, en materia medioambiental, de áreas verdes y espacios públicos; y iv) Instituciones: actores del sector público, locales y regionales; instituciones y documentos de regulación y gestión territorial específicos.

Para analizar las interacciones entre los componentes del modelo, a partir de la información recolectada en las entrevistas, se distinguen cuatro funciones elementales. Éstas cubren campos semánticos que ordenan las operaciones y vínculos que se realizan en el ecosistema urbano (relaciones inter-componentes) y que son señaladas a continuación: i) Adaptación (interacciones Comunidad/Ecosistema): 
usos, prácticas y rutinas de la comunidad en el ecosistema; impactos medioambientales en la comunidad; ii) Legitimación (interacciones Comunidad/Instituciones): mecanismos y estrategias de validación de las instituciones en la comunidad; procesos que influyen en el vínculo de la comunidad con los actores institucionales; iii) Planificación (interacciones Instituciones/ Entorno Construido): procesos de urbanización y mecanismos de regulación de las instituciones sobre el territorio; impactos del desarrollo urbano en los actores institucionales; y iv) Integración (interacciones Ecosistema/Entorno Construido): emergencia del ecosistema urbano; procesos que influyen en el desarrollo ambiental del ecosistema urbano. Las cuatro funciones teóricas forman una imagen del horizonte que persiguen las instituciones para conducir de manera sustentable el desarrollo del ecosistema urbano. Eso permite distinguir los puntos de encuentro entre la dimensión social y la dimensión ambiental del desarrollo sustentable, (Dempsey et al., 2011). De este modo, se reconocen las capacidades y limitantes institucionales para incorporar criterios ecológicos asociados al concepto de servicios ecosistémicos, (en sus vínculos y operaciones). Al introducir el concepto de servicios ecosistémicos en el modelo de análisis, se ponen a prueba las capacidades institucionales de incorporar una dimensión ecológica en los procesos de intervención territorial. Dentro de ese marco, se evalúa la capacidad de las instituciones y sus instrumentos de planificación, para incorporar criterios ecológicos en sus operaciones (Rusch et al., 2017; Dempsey et al., 2011).

\section{Resultados}

La construcción del modelo de análisis permite identificar los principales problemas sobre la vegetación urbana en Temuco y Padre Las Casas, relacionando cada componente a través de una función específica (figura 1). Las funciones se explican, como las tensiones dónde se ubican los nodos problemáticos de ambas ciudades a partir de los conceptos de: planificación, integración, adaptación y legitimación.

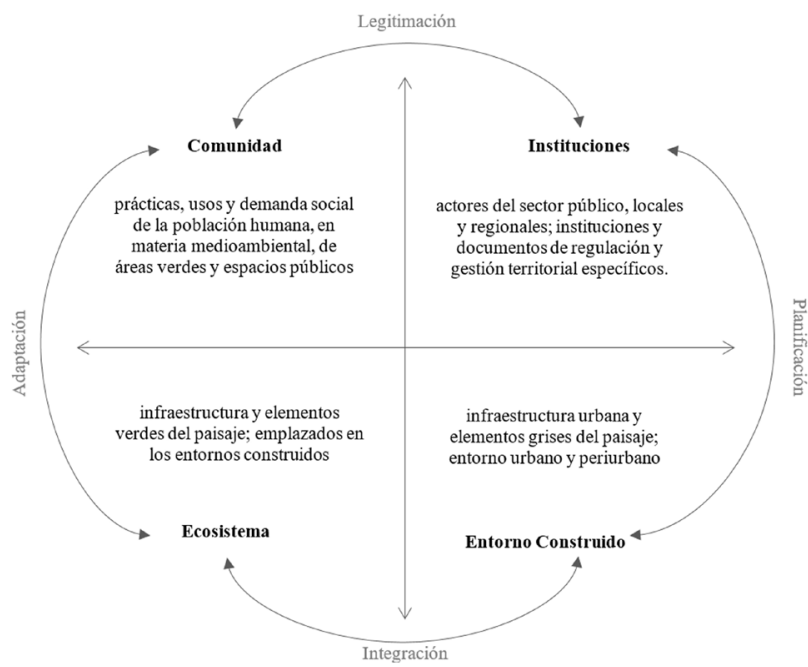

Figura 1. Modelo de análisis del ecosistema urbano en base a cuatro componentes y funciones elementales. Fuente: Elaboración propia (2017)

Figure 1. Analytic model of the urban ecosystem based on four elemental functions and components.

Source: Own elaboration (2017) 
El conocimiento a través de entrevistas sobre la red de actores institucionales que se involucran con temáticas de vegetación urbana, y sus desempeños como gestores del territorio, ha permitido distinguir las amenazas y oportunidades de cada organismo, sus capacidades de intervención y/o las debilidades que les impiden operar frente a la contingencia.

Se desglosan en la tabla 1 , los principales problemas de la vegetación urbana en ambas ciudades.

Tabla 1

Principales problemas de la vegetación urbana en Temuco y Padre Las Casas, por componente de análisis. Fuente: Elaboración Propia (2017)

Table 1

Analysis components of the main problems of urban vegetation in Temuco and Padre Las Casas. Source: Own elaboration (2017)

\begin{tabular}{|c|c|c|}
\hline & TEMUCO & PADRE LAS CASAS \\
\hline \multirow{6}{*}{ INSTITUCIONES } & $\begin{array}{l}\text { Funciones compartidas impiden la ejecución integral de } \\
\text { proyectos a nivel local. }\end{array}$ & Unidades técnicas no-especializadas en el territorio. \\
\hline & $\begin{array}{l}\text { Metas institucionales independientes y focalizadas, } \\
\text { fragmentan el desarrollo del ecosistema urbano. }\end{array}$ & $\begin{array}{l}\text { Metas institucionales independientes y focalizadas, } \\
\text { fragmentan el desarrollo del ecosistema urbano. }\end{array}$ \\
\hline & Centralización de normativas del territorio. & Intervención territorial descoordinada. \\
\hline & $\begin{array}{l}\text { Rendimiento político genera proyectos de corto plazo, } \\
\text { escasamente sustentables. }\end{array}$ & Prácticas clientelares en la entrega de cargos municipales. \\
\hline & Desconocimiento técnico sobre Silvicultura Urbana local. & Carencia de instrumentos ad-hoc a la intervención urbana. \\
\hline & $\begin{array}{l}\text { Establecen criterios para la incorporación de especies } \\
\text { nativas. }\end{array}$ & $\begin{array}{l}\text { Toma de decisiones orientada a la provisión de servicios } \\
\text { básicos y metas de corto plazo. }\end{array}$ \\
\hline \multirow{3}{*}{ COMUNIDAD } & $\begin{array}{l}\text { Fragmentación en la localización de las áreas verdes y } \\
\text { vegetación urbana. }\end{array}$ & $\begin{array}{l}\text { Fragmentación en la localización de las áreas verdes y } \\
\text { vegetación urbana. }\end{array}$ \\
\hline & $\begin{array}{l}\text { Desconocimiento de funciones de la vegetación y arbolado } \\
\text { urbano. }\end{array}$ & Estigmatización. \\
\hline & Valoración cultural de la vegetación urbana. & $\begin{array}{l}\text { No hay demanda de vegetación urbana y espacios públicos. } \\
\text { Demanda focalizada en espacios comunes y equipamiento. }\end{array}$ \\
\hline \multirow{5}{*}{$\begin{array}{l}\text { ENTORNO } \\
\text { CONSTRUIDO }\end{array}$} & $\begin{array}{l}\text { Dispositivos de seguridad y coerción del espacio público, } \\
\text { prioritarios a la vegetación urbana. }\end{array}$ & $\begin{array}{l}\text { Dispositivos de seguridad y coerción del espacio público, } \\
\text { prioritarios a la vegetación urbana }\end{array}$ \\
\hline & $\begin{array}{l}\text { Funciones compartidas impiden la ejecución integral de } \\
\text { proyectos a nivel local. }\end{array}$ & $\begin{array}{l}\text { Áreas verdes concentradas para mitigar la política } \\
\text { habitacional. }\end{array}$ \\
\hline & Centralización de normativas del territorio. & $\begin{array}{l}\text { Desarrollo urbano orientado a explotar el territorio, no a la } \\
\text { sustentabilidad. }\end{array}$ \\
\hline & $\begin{array}{l}\text { No consolidación de infraestructura verde en el ecosistema } \\
\text { urbano. }\end{array}$ & $\begin{array}{l}\text { Prioridad en seguridad pública y no en integración del } \\
\text { ecosistema urbano. }\end{array}$ \\
\hline & Contaminación acústica. & El tejido urbano no consolidado, aumenta los sitios eriazos. \\
\hline \multirow{4}{*}{ ECOSISTEMA } & Contaminación atmosférica (leña). & Contaminación atmosférica (leña y residuos). \\
\hline & No hay un plan de manejo de residuos. & No hay un plan de manejo de residuos. \\
\hline & Escasez hídrica. & Escasez hídrica. \\
\hline & Anegamiento. & Anegamiento. \\
\hline
\end{tabular}




\section{Principales hallazgos $\mathbf{y}$ problemas emergentes en la conurbación Temuco, Padre Las Casas}

Los municipios cuentan con una naturaleza jurídica dual: son organismos de administración del Estado y administraciones autónomas en atribuciones y competencia legal (Subsecretaría de Desarrollo Regional y Administrativo, 2008). Del mismo modo, sus instrumentos son sumamente estandarizados (Salazar, Irarrázabal y Fonck, 2016), convirtiéndose en un mecanismo que entorpece las dinámicas territoriales $\mathrm{y}$ urbanas. $\mathrm{El}$ desempeño de los municipios en relación al manejo de la vegetación urbana corresponde a una función privativa y dependiente de su administración, no obstante, la gestión local no es el único organismo que incide en el incremento, la disposición y la ubicación de los espacios públicos y verdes. Temuco, por ejemplo, en su componente institucional, enfrenta desafíos propios de una ciudad intermedia que, debido a su alza en la concentración de servicios y competencias económicas, sus acciones acogen hoy una demanda cívica más compleja y exigente, que ha reivindicado la importancia del arbolado público a partir de sus valoraciones culturales. En su proceso de legitimación, el municipio debe adecuar las exigencias ciudadanas al manejo del arbolado y la prioridad de recambio de especies exclusivamente nativas. Padre Las Casas en cambio, como administración local presenta desafíos de orden elementales. Su corta existencia institucional, que data de 1995 y la rápida expansión urbana, son dos factores que tensionan su capacidad de gestión para proveer servicios de calidad, desarrollándose un vínculo de legitimación hacia la comunidad de manera contingente, con el fin de resolver imprevistos y necesidades inmediatas. La comunidad presenta una gran cantidad de juntas vecinales, las que focalizan su demanda en espacios de reunión y organización como sedes sociales o equipamiento vinculado a servicios básicos (educación y salud), sin estar presente la exigencia de áreas verdes. En ambos municipios el desarrollo del entorno construido es desigual y opera según las intenciones de la promoción inmobiliaria. Temuco al ser ciudad capital y epicentro de la oferta en servicios y fuentes laborales, aumenta su plusvalía y calidad de vida, siendo condición de posibilidad para los habitantes que cuenten con el capital económico suficiente, en su establecimiento y permanencia. Padre Las Casas por otro lado, se desarrolla como la ciudad aledaña de Temuco y que desde sus orígenes se compone como un sector de la población que no logró integrarse a la ciudad capital. $\mathrm{Su}$ origen como municipio tiene relación con el sector inmobiliario para clases vulnerables y medias emergentes (políticas habitacionales promovidas por el gobierno central), debiendo asumir el costo del desarrollo de barrios fragmentados que carecen de elementos fundamentales en la planificación urbana: vías de circulación que distingan el tejido urbano, espacios públicos de calidad y equipamiento necesario para la entrega de servicios a su comunidad. Hasta ahora Temuco y Padre Las Casas parecen ser dos ciudades paradójicamente lejanas. Si bien son parte de una conurbación, su desarrollo administrativo, sus lógicas de legitimación y de crecimiento, abordan conflictos de interés en distintos niveles.

La vegetación urbana en ambos casos de estudio no es un tema prioritario, sin embargo, en Temuco se ha instalado en la agenda local como una oportunidad para mejorar el vínculo que existe con las comunidades. Esto se observa en la inversión de mejoras hacia espacios públicos, iniciativa municipal, y en la creación de nuevos parques urbanos para la ciudad (Eco Parque Corcolén, Temuco). Padre Las Casas aún se encuentra en un estado de desarrollo inicial y sus áreas verdes son los espacios remanentes de loteos de viviendas de construcción simultánea, con escasa disposición espacial y carencia de especies arbóreas. La estandarización de las políticas de urbanización, como la Ley 
General de Urbanismo y Construcciones (LGUC), contempla la disposición del suelo según el precio del mercado, sin atender a un estándar mínimo de espacio púbico y áreas verdes. Para mitigar el grado de complejidad de los instrumentos normativos de planificación urbana el Servicio de Vivienda y Urbanización (SERVIU) ha desarrollado y puesto a disposición 2 parques para la comuna. Los Parques Corvalán y Pulmahue son mecanismos de mitigación de áreas verdes para resolver la tensión de atender a los altos grados de concentración y centralización de las políticas habitacionales. No obstante, cabe rescatar que la ubicación de ambos parques no es estratégica, al estar ambos concentrados en el casco histórico de la comuna.

\section{Circuito Institucional de gestión de la vegetación urbana en Temuco, Padre Las Casas}

La gestión de la vegetación urbana en Temuco y Padre Las Casas depende de procesos sociales a nivel institucional; son el conjunto de actores y unidades administrativas que ejecutan programas y políticas, vinculándose de forma interdependiente, con distintos agentes para llevar a cabo una intervención en el territorio. A continuación, la organización del relato de los/las entrevistados/das, quienes describen sus capacidades de gestión sobre la vegetación urbana, según municipio. Esta lectura institucional comprende la sistematización de códigos emergentes en la conversación, tales como: recursos (normas y presupuestos), unidades (instituciones y sus actores), vinculaciones (capacidad de gestión $\mathrm{y}$ comunicaciones entre unidades y entre instituciones) y procesos (estrategias y líneas de acción). Todos estos elementos se combinan en las entrevistas, dando cuenta de una serie de acciones en el territorio que permiten identificar a los actores institucionales que llevan a cabo la implementación de una nueva área verde, la rehabilitación de un espacio público o el manejo de la vegetación de la ciudad. Esta lectura institucional se describe como la comprensión de un circuito o red institucional de gestión de la vegetación urbana, distinguiendo programas, unidades e instituciones. A continuación, se describen los programas, unidades e instituciones asociadas.

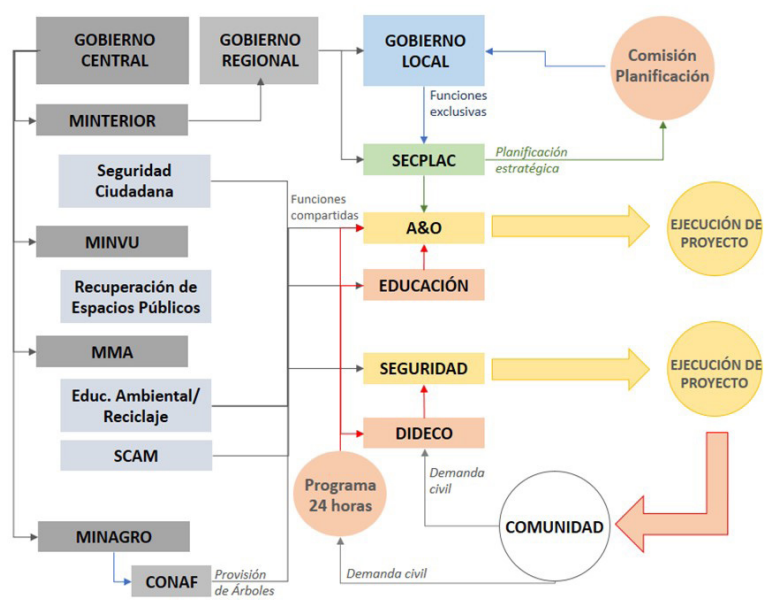

Figura 2. Circuito Institucional de gestión de la vegetación urbana: Caso Temuco. Fuente:

Elaboración propia (2017)

Figure 2. Institutional network of management urban vegetation: Temuco Case. Source: Own elaboration (2017) 
En la figura 2 es posible observar quiénes son los actores más relevantes en la gestión del manejo sobre las áreas verdes, el arbolado y los espacios público en la ciudad de Temuco, vinculando los estamentos que provienen del gobierno central, para el cumplimento de metas en su contraparte municipal y las unidades de la administración local, en la gestión del funcionamiento privativo de sus atribuciones. Este esquema ordena a los actores/instituciones, desglosando la lectura que realiza el sector público sobre el manejo de la vegetación en la comuna.

Como actor más relevante del circuito a nivel local, se identifica la Secretaría de Planificación (SECPLAN). Esta unidad trabaja vinculando redes estratégicas interunidades y entre instituciones externas a la administración local. Los profesionales disponen de altos grados de conocimiento técnico sobre su administración y son los encargados de identificar las problemáticas de base que dificultan la ejecución de las políticas públicas, para volverlas más expeditas. La secretaría asume un rol de facilitador en la institución entre los tomadores de decisiones y los profesionales técnicos, buscando medidas claras de prevención y planificación ante posibles contingencias.

Del mismo modo se caracterizan por ser profesionales de confianza, validados políticamente y a los cuáles se les delega gran parte de la toma de decisiones que en materias de expertos se requiere. Acá se desarrolla una agrupación de profesionales, descrita como la Comisión de Planificación, encargada de coordinar a la gestión local para llegar a acuerdos en la intervención, buscando coordinar de forma transversal los roles que cada unidad debe tomar a la hora de ejecutar un proyecto de áreas verdes o arbolado para la ciudad. Esta organización es una innovación llevada a cabo desde el año 2015 , cuyo objetivo es la evaluación del proyecto ciudad, a través de la revisión de proyectos inmobiliarios o la creación de nuevos espacios públicos, estableciéndose por primera vez en la historia municipal, la capacidad de coordinación a la hora de proyectar un espacio público con la opinión de todas las unidades que correspondan. Un ejemplo de ello es el criterio de revisión sobre proyectos sobre nuevas plazas y espacios públicos a disponer en el territorio, con el fin de conectarlas con otras ya establecidas, reduciendo la fragmentación territorial de la infraestructura verde y reduciendo costos de mantención posteriores. Buscar un aumento en el rendimiento de la mantención de un área verde en específico, pasa por anticipar dónde se crearán los nuevos conjuntos habitacionales o cuáles son las áreas prioritarias en la mantención de plazas, parques o plazoletas, localizando estos nuevos espacios públicos muy cercanos o inmediatos a lugares con similares características.

Temuco instala un énfasis en la gestión personalizada, respetando sus cargos históricos. El énfasis por el compromiso institucional es un motor que promueve el trabajo inter-unidades, siendo cada cargo validado por los tomadores de decisiones de forma diacrónica. Esta visión organizacional busca promover el compromiso y la experiencia curricular más que los conocimientos técnicos asociados a la ejecución de metas, lo que ha implicado la constante instalación de conocimientos específicos para el equipo de la unidad, cuyo objetivo es la modernización de la gestión de sus áreas verdes. Un ejemplo de modernización es el reciente Programa 24 Horas de la Dirección de Medio Ambiente, Aseo y Ornato, que apoya la gestión sobre el manejo de la vegetación urbana fortaleciendo la demanda civil a través de la educación ambiental, a la hora de intervenir los espacios de uso público. Este se lleva a cabo por medio de un asesor técnico municipal y un operador (asistente social), dupla que funciona como una alianza estratégica desde la mencionada dirección. El programa se dedica a solucionar las demandas de forma contingente que se presentan en el territorio, como una 
respuesta inmediata y cercana del vínculo municipio-comunidad, que está presente para legitimar los lazos de trabajo entre ambas partes.

Los organismos centrales, desde la política sectorial, se hacen presentes a través de los municipios, incorporando distintos programas de intervención. En el caso de los programas que maneja el Ministerio del Interior y Seguridad Pública, se desconcentra el programa Rehabilitación de Espacios Públicos y Seguridad Ciudadana, existiendo duplas de trabajo (asistentes sociales y arquitectos), dependientes de la Dirección de Seguridad Ciudadana del municipio, canalizando la intervención del programa con trabajo comunitario. Los criterios de intervención están sujetos a los diseños de espacios públicos establecidos por el nivel central, que constan de una serie de dispositivos para la provisión de seguridad al espacio público (luminarias tipo estadio, bancas estilo anti-vandálicas, máquinas de ejercicios).

El Ministerio del Medio Ambiente (MMA) realiza visitas a terreno hacia los establecimientos educativos de la comuna y busca de forma estratégica trabajar con el Programa 24 Horas del municipio. Sus intervenciones son puntuales, aisladas y no cuentan con una planificación, dificultad entre las instituciones para conocer los impactos de esta alianza a nivel pedagógico. Sin embargo, este esfuerzo institucional, acompañado por el sistema de certificación medio ambiental (SCAM), para obtener el sello verde ministerial, no contempla manejo de la vegetación urbana. Sus temáticas abordan el manejo de residuos y el cambio climático como objetivos de base para la institución.

El Ministerio de Vivienda y Urbanismo (MINVU), se incorpora como un actor estratégico para los espacios y plazas públicas a través de dos programas: Espacios Públicos y Programa Barrios. Ambos coordinan con SECPLAN, sin embargo, ninguno de ellos tiene una relación con la Dirección de Medio Ambiente, Aseo y Ornato. Las estrategias de estos programas no levantan como tema prioritario el manejo de la vegetación, no obstante, el Programa Barrios asegura iniciar una relación con la Corporación Nacional Forestal (CONAF), para la obtención de conocimiento sobre especies arbóreas pertinentes a establecer en lugares específicos y además, intercambiar conocimientos técnicos que se acerquen a hacia las comunidades en intervención.

Luego de la campaña Un árbol por un chileno (2013), CONAF debió ampliar su contexto de intervención incorporando su capacidad de gestión en el entorno construido. Cabe destacar que la poca articulación y estrategia de esta política pública, implicó la gestación de más de 17.000 millones de especies arbóreas exóticas ( $\sin$ la comprensión de su pertinencia con el territorio), y que no alcanzaron a llegar a un $20 \%$ en su establecimiento. Esta extensión de servicios tiene su continuidad en el 2014 con el programa Más árboles para Chile, siendo CONAF ahora una institución que debe incorporar elementos de la silvicultura urbana dentro de los códigos institucionales. A partir de la dinámica de provisión, CONAF toma un rol activo con las instituciones vinculadas a la vegetación urbana en Temuco, existiendo coordinación para el desarrollo de proyectos específicos. El circuito o red institucional identificada (figura 3) en la comuna Padre Las Casas, presenta actores relevantes dentro de la administración local, específicamente en sus tomadores de decisiones. La composición de su estructura organizacional en relación al desarrollo de áreas verdes, arbolado y espacios públicos se genera en la Dirección de Medio Ambiente, cuyos objetivos se enmarcan en la mantención de la vegetación, a través de operarios en el territorio. De manera complementaria, profesionales de la misma unidad, cumplen un rol de asesores comunitarios que se orientan en la participación de los agentes vecinales, por medio de iniciativas que estimulen 
la creación y ejecución de proyectos en barrios específicos. No es clara la visión y los objetivos de la dirección en cuanto a una mantención planificada de su vegetación, lo que responde a la construcción de un discurso entre sus actores, que ejecuta sus acciones frente a la contingencia de la ciudadanía, la que se expresa a través de demandas específicas: manejo de residuos, necesidad de poda en casos puntuales y necesidad de control frente a la gran cantidad de sitios eriazos que presenta el territorio. En este caso específico, SECPLAN responde a sus prioridades, instaladas en la actualización de sus instrumentos de planificación, específicamente el desarrollo de un nuevo Plan Regulador Comunal, cuyos objetivos se enmarcan en la construcción de una visión prospectiva ante la expansión de la ciudad, que se caracteriza por el desarrollo de proyectos inmobiliarios. Estas complejidades, han generado una ausencia en la coordinación de los equipos dentro del municipio, debiendo cada unidad referida a la construcción de espacios públicos, afrontar el desarrollo de proyectos bajo sus propias herramientas de gestión, con las instituciones derivadas de la política sectorial, llevando a cabo alianzas estratégicas de inversión a pequeña escala. La intervención, en tanto, se describe a través de proyectos puntuales, determinando acciones en vecindarios reconocidos como prioritarios para la orientación de la gestión barrial. La unidad que destaca en su desempeño como articulador entre las direcciones, se ejerce desde la Administración Municipal que, al contar con nuevos representantes, busca levantar un diagnóstico que dé cuenta sobre los principales desafíos y amenazas de la institución.

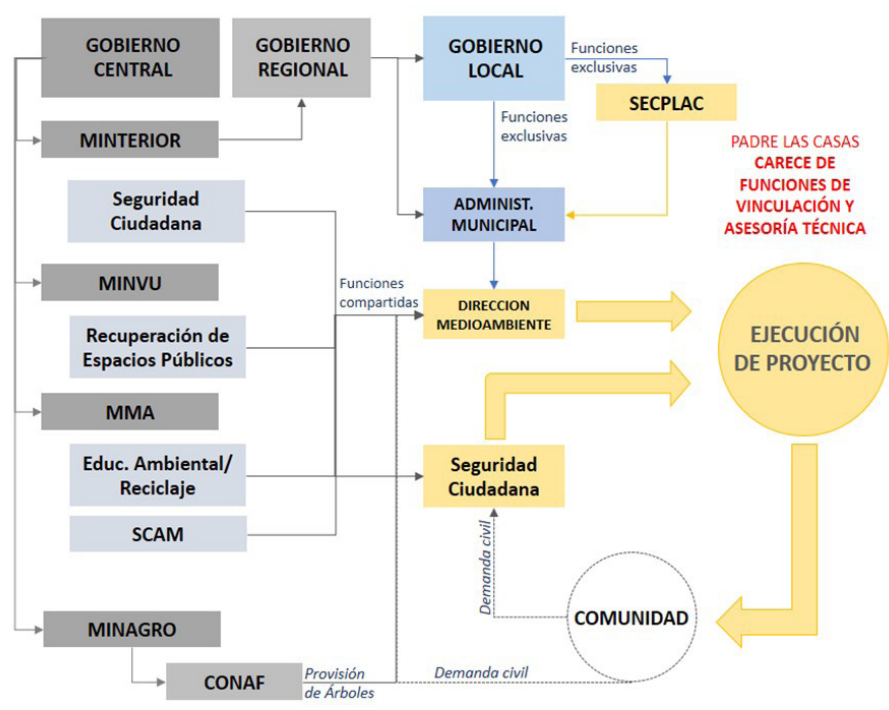

Figura 3. Circuito Institucional de gestión de la vegetación urbana: Caso Padre Las Casas. Fuente: Elaboración propia (2017)

Figure 3. Institutional network of management urban vegetation: Padre Las Casas. Source: Own elaboration (2017)

Las instituciones del gobierno central que se instalan en el territorio refieren a la entrega de servicios enfocados hacia la ciudadanía, presentando estrategias de acompañamiento para los sectores más vulnerables. En el caso de la Dirección de Seguridad 
Ciudadana, el programa que opera con recursos desde el nivel central, establece sus lineamientos de acción en relación al acompañamiento de jóvenes en situación de riesgo, buscando construir mecanismos de control frente al consumo problemático y la delincuencia, estableciendo la inversión de sus fondos en actividades lideradas por el acompañamiento social, careciendo del desarrollo de intervenciones en los lugares de convivencia.

MINVU en cambio, se manifiesta como un actor relevante que ha instalado tres ejes de intervención, por medio de las siguientes estrategias:

El desarrollo de proyectos inmobiliarios para sectores vulnerables y medios, bajo sus programas y subsidios habitacionales (D.S 49, D.S 105 y D.S 1). En este sentido, la entrega de nuevos barrios, cuya tipología prepondera en la construcción de viviendas de baja densidad, han permitido la creación de áreas verdes públicas, que tienden al aumento y desafían los escasos recursos de la Dirección de Medio Ambiente, para asumir nuevos costos de mantención.

El Programa Barrios, como el líder en la rehabilitación de los espacios públicos que presentan mayores deficiencias, siendo lugares de inseguridad en distintos puntos de la ciudad. Sus estrategias, apuntan al trabajo colectivo de los primeros vecindarios emplazados en el territorio, disponiendo de recursos para el mejoramiento físico del espacio, la implementación de diseños participativos, y el aumento de áreas verdes en las unidades vecinales de mayor fragmentación.

La creación de parques urbanos para la ciudad, Parque Pulmahue y Corvalán, se ubican en el casco histórico de la reciente comuna, apuntando a ser los grandes espacios verdes de Padre Las Casas. No obstante, las brechas comunicacionales con el municipio, y la carencia de recursos de la unidad local, entorpecen el buen manejo de ambas áreas verdes, cuyas especies establecidas en el territorio, ya se encuentran secas y sin reparación.

El carente presupuesto municipal, asociado también a su incipiente estadio organizacional, entorpece los avances en materias de espacios públicos de calidad. Sin embargo, la oportunidad de contar con profesionales de alta capacidad de gestión hace posible la valoración por los temas ambientales y la incorporación de nuevos enfoques frente a la mantención de estos espacios. Un ejemplo de ello es la presencia de fondos del MMA, como el Fondo de Protección Ambiental, que liderados por los profesionales del equipo de Medio Ambiente de la comuna, buscan vincular a las juntas vecinales en el desarrollo de áreas verdes, para implementar estrategias de mantención que incluyan a los vecinos. Estas consideraciones elaboradas desde la dirección medio ambiental, buscan ser estrategias que reduzcan los gastos asociados a la mantención de áreas verdes, desde el municipio, delegando dicha ejecución a la comunidad. La participación de otros actores como CONAF, no se observan como determinante en el incremento del arbolado público, siendo éste un tema que se identifica pendiente por parte del equipo municipal.

Padre Las Casas da cuenta de una explosión inmobiliaria que ha generado nuevos espacios verdes para la ciudad, asumiendo el costo de la política urbana nacional, que para estos fines, delega el incremento de dichas áreas a la gestión local, sin una mitigación por el aumento de costos y de recursos, a invertir para la futura gestión de los lugares de convivencia establecidos.

\section{Discusión}

Brechas y oportunidades en la gestión de la vegetación urbana en Temuco y Padre Las Casas

Los gobiernos locales en Chile funcionan como los organismos autónomos que han de administrar territorios específicos, entregar 
una calidad de servicios óptimos y colaborar en la entrega de beneficios que el Estado les comparte y delega a través de convenios y programas. El interés de este documento es complementar los trabajos elaborados en esta materia, sobre las problemáticas que se viven en el territorio, y que presentan una escasa reflexión sobre las unidades de la administración local (Salazar et al., 2016). En este sentido, los municipios juegan un doble rol a nivel de organización: realizan acciones exclusivas de su competencia, del mismo modo que han de acomodarse en la entrega de servicios que están sujetos a las decisiones del gobierno central.

\section{Temuco}

Actualmente, la alta confluencia territorial de estamentos que se ubican en la comuna y la historia de su organización municipal, han sido facilitadores para desarrollar vínculos comunicacionales, tanto al interior del organismo local, como en su relación con las instituciones de orden nacional. La alta coordinación estratégica a nivel interno en el municipio da resultados explícitos al momento de establecer una mesa de comunicación transversal entre directores y asesores, frente a la elaboración de cada proyecto que tiene repercusiones en el territorio. Esta Mesa Territorial, busca establecer la comunicación entre las unidades de la organización, y definir qué rol tomará cada unidad para la mediación y negociación con los organismos públicos y privados, que se vean comprometidos en la intervención. Por ejemplo, antes de esta instancia, si existía un proyecto de rehabilitación del espacio público, la conversación se realizaba sólo en la Dirección de Seguridad, sin buscar información técnica con el resto de las unidades municipales, para otorgarle una funcionalidad adecuada al espacio, por sobre la estética.

Los actores del municipio, en este sentido, buscan instalar un espacio de reflexión sobre la pertinencia de los proyectos, tanto habitacionales, de espacios públicos o comerciales, siendo la administración local, la que deba coordinarse para que los diferentes asesores técnicos (de cada unidad), provean de información a la hora de evaluar pertinencia y sustentabilidad de dicha intervención. Ahora, si ha de ejecutarse un proyecto para la ciudad desde la Dirección de Seguridad, los agentes de esta unidad han de informar sobre la iniciativa y del mismo modo coordinarse con otras unidades a la hora de la evaluación del proyecto (Unidad de Ornato, SECPLAC, entre otros). Estas nuevas operaciones están en constantes ajustes, cambios que sufre la administración en sus profesionales, demandas sociales contingentes $o$ las mismas presiones políticas que alteran el orden de la administración.

La Comisión de Planificación es una instancia municipal que establece una nueva visión organizacional, que intenta reunir las capacidades técnicas de las distintas unidades, para establecer roles y aportes de orden transversal. En este sentido, los operadores y ejecutivos de proyectos que se vinculan con el territorio asumen tareas complejas. Por un lado, las unidades administrativas se coordinan para responder como revisores de proyectos de interés privado o provenientes del sector público, y por el otro, buscan un mayor alcance en su vinculación con la comunidad; por ejemplo, encontrar a profesionales de trabajo social en la Dirección Medio Ambiente, Aseo y Ornato, cuyo objetivo es resolver temas como la educación ambiental, entrega de información sobre las responsabilidades en la mantención de las áreas verdes de uso público, entre otros relativos al cuidado del espacio público.

En la comprensión del funcionamiento entre instituciones, el cumplimiento de objetivos e intervenciones siempre suele ser más complejo. No sólo depende del estado y las características de cada estamento, sino que también de los intereses políticos que han de rendir resultados claros hacia los objetivos 
que cada institución plantea. A modo de ejemplo, un primer intento se da de forma compartida en el vínculo que ha debido generar CONAF, con cada institución que se vincula con la creación, incremento o rehabilitación de espacios públicos. Tanto MINVU en su Programa Barrios, como la Dirección de Medio Ambiente, Aseo y Ornato del municipio, aseveran haber iniciado una relación con la corporación, buscando instalar criterios comunes a la hora de intervenir la vegetación urbana de la ciudad. Estos son nuevos mecanismos de acción, indicadores favorables frente a la coordinación entre instituciones, que se encuentran en una etapa inicial de creación de objetivos y criterios.

Estos ejemplos se traducen en procesos de mejoramiento continuo para el liderazgo de la gestión local y la gobernanza estratégica de toda institución instalada en el territorio. Estas capacidades aún se encuentran en disputa, con la histórica estandarización que cada servicio público presenta en su carácter más ontológico, sin embargo, las presiones globales por la entrega de servicios de calidad implican la creación de nuevos perfiles de competencia en la generación y en la gestión de los proyectos. Los altos grados de urbanización, de bienes y servicios que ofrece la capital regional, facilitan el acceso del municipio hacia las redes inter-institucionales de forma estratégica y del mismo modo, instala canales de comunicación expeditos e incluso informales, al utilizar los mismos espacios céntricos de la ciudad para ejercer sus actividades. No obstante, las últimas crisis económicas asociadas al fenómeno de la globalización, es un aspecto que ha presionado el desarrollo, sobre todo a los municipios de tamaño intermedio, que indagan en nuevas estrategias de competitividad para mitigar el impacto económico en la población. La incorporación de una forma de gobernabilidad corporativa, asociada a una lógica empresarial en la gestión y ejecución de las políticas públicas, han implicado un proceso de individuación entre los agentes que intervienen en el territorio, produciendo una ruptura en la comunicación en las instituciones que desarrollan metas y objetivos anuales. Este estado de individuación en la planificación y ejecución de proyectos para la ciudad, implican muchas veces la doble intervención en el territorio y de manera consecutiva, el reflejo de estas intervenciones en el paisaje de la ciudad.

La visión prospectiva que ha logrado comenzar a desarrollar la ciudad de Temuco y que toma sentido a través de su experiencia organizacional, busca resolver los problemas consecuentes de su primera etapa de urbanización. La posibilidad de contar con una alta gama de servicios que abastecen las necesidades de la población ha complejizado las problemáticas y a su vez, los objetivos que ha de plantearse la administración local a través de sus planes indicativos y normativos (Precht, Reyes, Salamanca, 2017). Son objetivos y acciones concretas las que apuntan a buscar una ciudad más sustentable, como plantear la observación específica y pertinente del territorio a través de sus recursos naturales, buscando pactar la deuda histórica que presenta el entorno construido, a partir de sus problemas ambientales. Las duras consecuencias en temas de escasez hídrica y de contaminación, han sido la oportunidad en la gestión local para replantearse nuevas alianzas estratégicas con otros organismos y desarrollar otras metodologías de planificación.

\section{Padre Las Casas}

En la actualidad, los municipios enfrentan escenarios altamente cambiantes. Los más frecuentes están dados por las presiones hacia la modernización de sus orgánicas, la distribución de los poderes económicos en el territorio y su frágil relación con la comunidad. En el caso de Padre Las Casas todos estos factores aumentan debido al reciente origen de la institución.

Más allá de su carácter residencial, la 
comuna no cuenta con una confluencia de servicios públicos regionales de gran envergadura: plaza cívica y tejido urbano que conecte la ciudad. Es por lo tanto carente en la observación de arbolado urbano y cuenta con parques de accesibilidad reducida, ya que se emplazan alrededor de unidades habitacionales específicas, que no se conectan con vías troncales. La baja influencia de las instituciones en el territorio da cuenta de una gestión local incipiente y que aún no aborda estrategias comunicacionales al interior de su organización. De esta manera se vuelve exclusiva la acción unívoca de la deliberación por parte de los directivos u otros agentes de misma jerarquía institucional, decretando acciones no sólo en ámbitos políticos (exclusivos de sus cargos), sino que también en materias técnicas, estratégicas y de planificación.

El resultado de estas prácticas no sólo implica la carencia de actores específicos que han de estar presentes en la orgánica local, sino que también deslegitima las acciones políticas, alejando al municipio en su vínculo con la comunidad. La estrecha y vertical relación que tienen los tomadores de decisiones sobre lo que ocurre en el territorio, reduce las capacidades de gobernanza y de alcance de metas hacia el largo plazo, para considerar desde la capacidad institucional un proyecto de ciudad. Las acciones se consideran para la resolución de conflictos territoriales emergentes, y que en su mayoría no se vinculan con el apoyo de otras instituciones del Estado en la asistencia técnica de proyectos e intervenciones.

La reciente consolidación de la administración en el territorio y su escasa experiencia municipal, implican el desarrollo de una gestión que resuelve problemáticas de base para la población (tareas mínimas y contingentes). Esta dinámica sujeta a la necesidad de vivienda muestra lo que es Padre Las Casas en la actualidad: un lugar de suburbanización amplio, con escasos servicios para la comunidad, validando su perfil como ciudad-dormitorio. Sus capacidades de gobernabilidad, denotan una problemática consecuente de su corta vida como gestión local. Su paisaje urbano refleja una visión de gestión local empresarial, que a través de propuestas habitacionales estandarizadas, logra fijar capitales en el territorio con empresas inmobiliarias que monopolizan la oferta habitacional a escala local.

La brecha entre las demandas de la comunidad y los recursos públicos para responder a esas demandas ha generado una política pública contingente desde la gestión local y sectorial. En tal caso, la vegetación urbana es un elemento que se incluye en la conformación de la ciudad, cumpliendo una función estética del paisaje, sin ser incluido en los objetivos y metas municipales prioritarias. La ausencia de visiones prospectivas para desarrollar ciudades sustentables, transforman el espacio local e impactan en la calidad de vida de sus residentes y usuarios (Orellana, Allard, Néspolo y Mercado, 2012).

Dentro del circuito de análisis institucional se observan actores técnicos que pueden asesorar la ejecución de acciones y programas con la comunidad. No obstante, la falta de una planificación con objetivos concretos y con una visión a largo plazo, impide que el municipio establezca una estrategia que le permita aprovechar las inversiones que ingresan, como proyectos de vegetación urbana.

\section{Conclusiones}

Instalar el discurso para llevar a cabo decisiones $y$ acciones hacia ciudades sustentables, implica en nuestras ciudades intermedias chilenas, elaborar nuevas estrategias que puedan lidiar con los problemas estructurales que aún persisten en el territorio. Esta visión supone fórmulas concretas y escenarios que permitan determinar el vínculo que se 
ha formado entre el entorno construido y el ecosistema y de qué manera la planificación es una herramienta efectiva para organizar el territorio. Del mismo modo, los procesos de urbanización han hecho efectiva la consolidación del modelo de ciudad dispersa, levantando problemáticas de segregación importantes. La capacidad de las instituciones se encuentra en la visión prospectiva, para lograr de manera organizada, sistemática y comprometida (alianzas estratégicas), evitar el solapamiento de intervenciones en el territorio, con el objetivo de orientar las metas inter-institucionales, en el desarrollo armónico de las ciudades de Chile

La planificación a escala micro-territorial comprende la expresión directa de los gobiernos locales en sus territorios. Sin embargo, la función del gobierno central es facultar de manera gradual, la capacidad de autogestión y deliberación en los gobiernos locales. Del mismo modo, la limitación de recursos de los municipios y su dependencia hacia el aparato central reduce que los gobiernos locales puedan liderar su capacidad de desarrollo endógeno.

\section{Referencias}

Balvanera, P. \& Cotler, H. (2007). Acercamientos al estudio de los servicios ecosistémicos. Gaceta ecológica, 84-85, 8-15. Recuperado de http://www.redalyc. org/articulo.oa? $\mathrm{id}=53908502$

Caro-Caro, C. \& Torres-Mora, M. (2015). Servicios ecosistémicos como soporte para la gestión de sistemas socioecológicos: aplicación en agroecosistemas. Orinoquia, 19 (2), 237-252. http://dx.doi. org/10.22579/20112629.338

Charmaz, K. (2006). Constructing Grounded Theory: a practical guide. London: SAGE Publications.
Dempsey, N., Bramley, G., Power, S., \& Brown, C. (2011). The social dimension of sustainable development: defining urban social sustainability. Sustainable Development, 19(5), 289-300. https://doi. org/10.1002/sd.417

Flores, R. (2009). Observando observadores: una introducción a las técnicas de investigación social. Santiago de Chile: Ediciones Universidad Católica. Recuperado de http://www.jstor.org/ stable/j.ctt17t76p7

Glaser, B. \& Strauss, A. (1967). The discovery of grounded theory. Chicago: Aldine Press. ISBN: 0-202-30260-1

Glaser, B.G. (2002). Conceptualization: on theory and theorizing using grounded theory. International Journal of Qualitative Methods, 1(2). Recuperado de https://sites. ualberta.ca/ iiqm/backissues/1_2Final/pdf/ glaser.pdf

Gómez-Baggethun, E., Gren, Å., Barton, D.N., Langemeyer, J., McPhearson, T., O'Farrell, P., ... Kremer, P. (2013) Urban Ecosystem Services. In Urbanization, Biodiversity and Ecosystem Services: Challenges and Opportunities (pp. 175251). Springer International Publishing

Grimm, N., Grove, M., Pickett, S., \& Redman, C. (2000). Integrated approaches to Long-term studies of urban ecological ecosystems. BioScience, 50 (7), 571$584 . \quad$ https://doi.org/10.1641/00063568(2000)050[0571:IATLTO]2.0.CO;2

Laterra, P., Martín-López, B., Mastrángelo, M., \& Garibaldi, L. (2017). Servicios ecosistémicos en Latinoamérica. De la investigación a la acción. Ecología Austral, 27(1-bis), 094-098. https://doi. org/10.25260/EA.17.27.1.1.611 
Orellana, A., Allard, P., Néspolo, R., \& Mercado, J. (2012) Gestión urbana municipal a escala metropolitana: modelos en competencia. Revista Geografia Norte Grande, 51, 67-80. http://dx.doi. org/10.4067/S0718-34022012000100004

Padilla Beltrán, J., Vega Rojas, P., \& Rincón Caballero, D. (2014). Teoría fundamentada y sus implicaciones en investigación educativa: el caso de Atlas.ti. Revista de Investigaciones UNAD, 13(1), 23-39. http:// dx.doi.org/10.22490/25391887.1129

Precht, A., Reyes, S., \& Salamanca, C. (2017). El ordenamiento territorial en Chile. Santiago de Chile: Ediciones Universidad Católica. ISBN: 978-956-142008-3

Rusch, V., Rusch, G., Goijman, A., Varela, S., \& Claps, L. (2017). Los servicios ecosistémicos como soporte para la toma de decisiones ambiental y socialmente sustentables. Ecología Austral, 27(1bis), 162-176. https://doi.org/10.25260/ EA.17.27.1.1.295

Salazar, G. Irarrázabal, F., \& Fonck, M. (2016). Ciudades intermedias y gobiernos locales: desfases en la Región de la Araucanía, Chile. EURE (Santiago), 43(130). https://doi.org/10.4067/s025071612017000300161
Strauss, A. \& Corbin, J. (2002). Bases de la investigación cualitativa. Técnicas y procedimientos para desarrollar la teoría fundamentada. Bogotá. Colombia. CONTUS. Editorial Universidad de Antioquia. 341 p. ISBN 9586556247

Subsecretaría de Desarrollo Regional y Administrativo (2008). Manual de Gestión Municipal. Recuperado de http:// www.subdere.cl/documentacion/manualde-gestion-municipal-actualizado-ala\%C3\%B1o-2008-0

Trinidad-Requena, A., Carrero-Planes, V., \& Soriano, R.M. (2006). Teoría Fundamentada "Grounded Theory". La construcción de la teoría a través del análisis interpretacional. Cuadernos metodológicos, 37. Madrid: Centro de Investigaciones Sociológicas. ISBN: 84-7476-398-3

Vásquez, A. (2016). Infraestructura verde, servicios ecosistémicos y sus aportes para enfrentar el cambio climático en ciudades: el caso del corredor ribereño del río Mapocho en Santiago de Chile. Revista de Geografia Norte Grande, 63,63-86. http://dx.doi. org/10.4067/S0718-34022016000100005 